DOI: https://doi.org/10.15407/techned2018.05 $: \underline{080}$

\title{
SPEEDS AND TORQUES RELATIONS OF THE TWO-MOTOR ELECTRIC DRIVE WITH GEARLESS ELECTROMECHANICAL DIFFERENTIAL
}

Journal

Publisher

ISSN

Issue

Pages
Tekhnichna elektrodynamika

Institute of Electrodynamics National Academy of Science of Ukraine 1607-7970 (print), 2218-1903 (online)

No 5, 2018 (September/October)

$80-83$

\section{Authors}

\section{V.P. Styazhkin ${ }^{1 *}$, V.I. Teriaiev², S.I. Gavryluk ${ }^{1}$}

1 - Institute of Electrodynamics National Academy of Sciences of Ukraine,

pr. Peremohy, 56, Kyiv, 03057, Ukraine,

e-mail: tems@ukr.net

2 - National Technical University of Ukraine "Igor Sikorsky Kyiv polytechnic institute",

pr. Peremohy, 37, Kyiv, 03056, Ukraine,

e-mail: kpivit@gmail.com

* ORCID ID : http://orcid.org/0000-0003-0602-1112

The speeds and torques relationship of two-motor electric drive with a gearless electromechanical differential in a closed two-channel speed control system is established. The static and dynamic modes of operation of the system are considered. References 5, figures 4. 
Key words: two-motor electric drive, gearless electromechanical differential, mathematical model, two-channel control system.

Received: 02.03 .2018

Accepted: 11.05 .2018

Published: 16.08 .2018

\section{References}

1. Teriaiev V.I., Styazhkin V.P., Gavryluk S.I. The two-channel adjustable electric drive. Patent UA No 124134, 2017. (Ukr)

2. Chemodanov B.K. Servo drive in three volumes. Moskva: Moskovskii gosudarstvennyi tekhnicheskii universitet imeni N.E. Baumana, 1999. 903 p. (Rus)

3. Basharin A.V., Novikov V.A., Sokolovskij G.G. Control of electric drives. Leningrad:

Energoizdat, 1982. 392 p. (Rus)

4. Klepikov V.B., Khudiaiev A.A., Polenok V.V. Iterative two-channel servodrive of feed high precision machine tools and mechanisms. Tekhnichna Elektrodynamika. 2015. No 5. Pp. 26-35. (Ukr)

5. Xiao J.R., Pan J.F. Position tracking control of two permanent magnet linear synchronous motors. International Conference on Power Electronics Systems and Applications - Smart Mobility , Power Transfer \& Security (PESA). 2017. Hong Kong, China. December 12-14, 2017. Pp. 1-4. DOI: http://dx.doi.org/10.1109/PESA.2017.8277729 